\title{
Bladder Metastasis from Breast Cancer: A Systematic Review
}

\author{
Uday Karjol $^{1}$, Pavan Jonnada ${ }^{1}$, Sushma Cherukuru ${ }^{2}$, Ajay Chandranath ${ }^{1}$ \\ 1. Surgical Oncology, Kidwai Memorial Institute of Oncology, Bangalore, IND 2. Pathology, AMPATH Laboratories, \\ Hyderabad, IND
}

Corresponding author: Pavan Jonnada, pavan.j.1903@gmail.com

\begin{abstract}
Breast cancer is the leading cause of cancer death in women, and most breast cancer related deaths are due to metastasis. Urinary bladder metastasis from breast cancer is rarely reported in the literature. In this review, we examined the reported cases of breast cancer metastasizing to the urinary bladder, with the objective of identifying clues that could help physicians in diagnosing and planning further treatment. We performed a systematic review of the literature to analyze the clinical and pathological profile of this disease. We thoroughly examined and systematically reported data regarding epidemiology, the pattern of spread, signs and symptoms, pathology and hormonal status, diagnostic workup, management, and outcomes. Urinary bladder metastases from breast cancers are more common in invasive lobular carcinoma. In addition to asymptomatic presentations, most cases present with hematuria and voiding dysfunction. This review summarizes the insights into the incidence, clinical presentation, diagnostic workup, management, and prognosis of urinary bladder metastasis in patients with breast cancer.
\end{abstract}

Received 03/11/2020 Review began 03/19/2020 Review ended 03/19/2020 Published 03/25/2020

() Copyright 2020 Karjol et al. This is an open access article distributed under the terms of the Creative Commons Attribution License CC-BY 4.0., which permits unrestricted use, distribution, and reproduction in any medium, provided the original author and source are credited.
Categories: Urology, General Surgery, Oncology

Keywords: bladder metastasis, breast cancer, oncology, breast neoplasm, urinary bladder neoplasms, distant metastasis

\section{Introduction And Background}

Breast cancer is the most common cancer, accounting for almost one in four cancer cases among women [1]. The incidence of breast cancer is highest in Australia, Europe, and North America. Mortality due to breast cancer is highest in Fiji [1]. Despite increased awareness, screening programs, and advancements in treatment, breast cancer remains the leading cause of cancer death in women. Most breast cancer related deaths are due to metastasis. Urinary bladder metastasis from breast cancer is a real phenomenon but rarely reported in the literature.

In this review, we examined the reported cases of breast cancer metastasizing to the urinary bladder to identify clues that could help physicians in diagnosing and planning further treatment. We electronically searched PubMed, Medline, and Google Scholar databases for articles published between 1950 and 2019 using the following keywords: "bladder metastasis”, "breast cancer”, “oncology”, "breast neoplasm”, "urinary bladder neoplasms", and "distant metastasis". All case reports, case series, and review articles were thoroughly examined, and data regarding epidemiology, the pattern of spread, signs and symptoms, pathology and hormonal status, diagnostic workup, management, and outcomes were systematically reported. The PRISMA (Preferred Reporting Items for Systematic Reviews and Meta-Analyses) flow chart is shown in Figure 1. 


\section{Cureus}
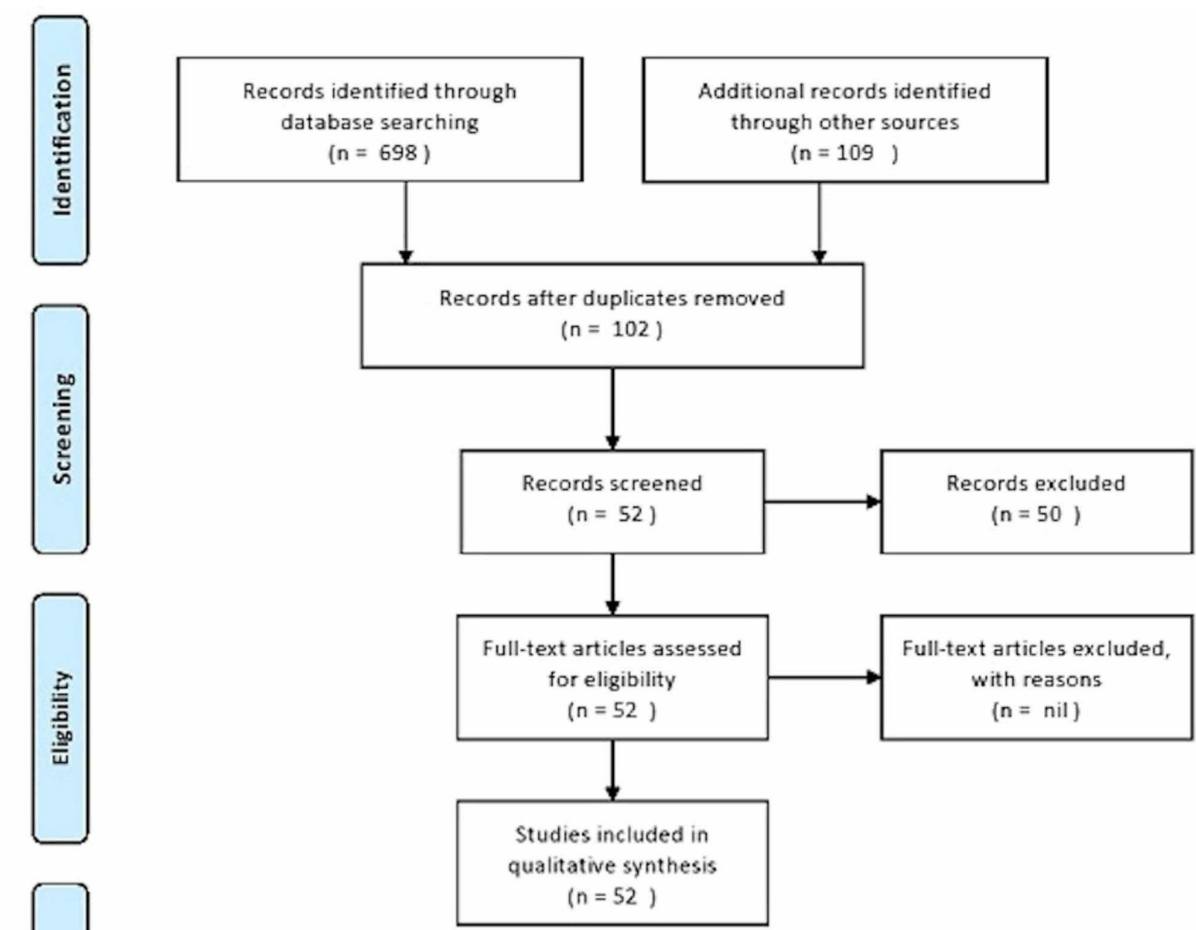

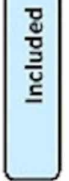

\section{FIGURE 1: PRISMA flow chart}

PRISMA, Preferred Reporting Items for Systematic Reviews and Meta-Analyses

\section{Review}

Though rare, secondary tumors of the urinary bladder can present a diagnostic dilemma in considering a differential diagnosis of primary bladder cancer. They comprise less than $2 \%$ of all bladder cancers [2]. The most common primary sources are the stomach, breast, colon cancer, and melanoma [2]. Most of these secondary tumors are because of direct extension from another pelvic neoplasm, such as sigmoid, prostate, or cervical cancer. Metastases from distant organs are sporadically reported in the literature, with the most common being the stomach, lung, and melanoma [3].

Approximately $45 \%$ of breast cancers present with metastasis, which can occur in almost any organ. Breast cancer frequently metastasizes to the bone, lung, liver, and brain, known as organ tropism [4]. For patients with metastatic breast cancer (MBC), 30\% to $60 \%$ have lesions in the bone, $4 \%$ to $10 \%$ in the brain, $15 \%$ to $32 \%$ in the liver, and $21 \%$ to $32 \%$ in the lung [5]. Urinary bladder metastases (UBMs) are rare and reported in the literature occasionally.

UBMs from breast cancer are extremely rare, with about 65 cases reported in the literature. Autopsy studies analyzing UBM from breast cancer reported the incidence ranging from none to almost 7\%. In 1950, in a monumental analysis of 1,000 autopsies, Abrams et al. identified four (2\%) cases of UBM after reviewing 167 cases of MBC [6]. In 1951, Klinger identified three (<1\%) cases of UBM from MBC after reviewing 5,000 autopsy cases of genitourinary tract metastases [7]. Ganem and Batal surveyed the published autopsy reports through 1956 and found 16 cases of UBM [8]. In 1967, Goldstein identified four cases of UBM (1.2\%) from MBC after reviewing 341 autopsy cases of patients who died of advanced breast cancer [9]. Pontes and Oldford reported approximately $7 \%$ of UBM among 85 autopsies [10].

The pattern of metastasis of breast cancer may be related to the histological type of cancer [11]. The major pathological subtypes of breast cancer are ductal and lobular carcinoma. Invasive ductal carcinoma (IDC) is the most common subtype of breast cancer, accounting for $90 \%$ of cases [12]. Invasive lobular carcinoma (ILC) is the second most common subtype, accounting for $8 \%$ to $14 \%$ of cases [13]. UBMs from breast cancer are more common in ILC than IDC. The metastatic pattern of ILC differs from IDC in that it tends to occur as a diffuse thickening of mucosa rather than a discrete nodule. It has a higher propensity to metastasize to 
serosal surfaces (gynecological and gastrointestinal tracts), and the spread from these sites may be the cause for the greater incidence of bladder involvement [11,14,15]. Feldman et al. noted that one-third of patients with UBM had ILC [11]. Borst and Ingold noted that 3\% of ILC metastasized to peritoneum-retroperitoneum compared with $0.6 \%$ of IDC [13].

The pattern of spread to UBM from breast cancer is because of tumor embolus that does not seed in the lung but passes through pulmonary circulation and reaches the target organ and soil there, causing a metastasis [8-10]. Pontes and Oldford postulated that breast cancer metastasizes to the bladder through retroperitoneal involvement [10]. Patients receiving steroids may develop metastasis at unusual sites due to the possible influence of the immunosuppressive effect of steroids [16].

Based on gene expression profiles and receptor status (estrogen receptor [ER], progesterone receptor [PR], human epidermal growth factor receptor 2 [HER2]) and on proliferation status as assessed by Ki67, Perou et al. subdivided breast cancer into four main clinical subtypes [17]: luminal A (ER+/PR+), luminal B (ER+/PR+/HER2-/+/Ki67+), HER2 overexpressing (ER-/PR-/HER+), and basal-like/triple-negative (TN) (ER-/PR-/HER2-). The expression of ER and PR in the UBM is expected to be similar to that in primary breast cancer. However, discordant expression of the ER and PR status in primary breast cancer and metastatic bladder lesions have been observed in studies $[11,18,19]$. Iguchi et al. hypothesized that the heterogenous expression of ER and PR between the primary tumor and metastatic lesion is based on cell clonality; breast cancer cells are polygonal. Besides clonal theory, change of expression can occur after adjuvant therapy due to the elimination and growth of PR-positive or ER-negative cells or due to gene mutations [20].

Synchronous and metachronous presentations are observed for UBM from breast cancer [21,22]. The majority of UBMs present as a part of widespread disease. Solitary metastasis to the urinary bladder without evidence of any other distant disease has been sporadically reported [11,14,19,23]. Clinical presentation can range from asymptomatic presentation to gross hematuria, obstructive uropathy, and renal failure $[11,19,24,25]$. The most common presenting symptom is painless gross hematuria. Gross hematuria with a history of breast cancer should be carefully investigated, considering the side effects of cyclophosphamide as a treatment of primary breast cancer, regardless of time or duration of treatment. Feldman et al. reported in their study that 17 of 19 patients reviewed presented with urinary symptoms of frequency, nocturia, incontinence, dysuria, microscopic hematuria, and back pain [11]. Detrusor involvement can lead to irritative voiding symptoms and can present earlier than hematuria. Balachandran and Duckett reported in their study that their patient presented with urgency and nocturia and that urodynamic testing revealed overactivity of the detrusor [26]. Soon et al. reported that UBMs were detected before the diagnosis of breast cancer and concluded that urinary incontinence might be the first sign of cancer; therefore, a careful evaluation for the possibility of UBM should be sought in patients with a history of breast cancer and urinary symptoms [21]. Focused ultrasonography of the urinary bladder is the first-line diagnostic investigation for a patient of breast cancer with voiding symptoms. The critical diagnostic modalities during workup for suspicious UBM are cystoscopy and biopsy. Cystoscopy is useful in identifying the disease and can aid resection. However, random biopsies taken in standard cystoscopy can reveal UBM [11,26,27]. Contrast-enhanced CT becomes valuable when there is evidence of renal impairment, suggesting obstructive nephropathy or equivocal cystoscopy [28]. Fluorodeoxyglucose positron emission tomography can improve the prediction of the clinical outcome of previously treated patients and improve the imaging evaluation of bladder neoplasm [29].

In our opinion, due to varied presentations of UBM from breast cancer, we suggest that biopsies should be undertaken in a patient suggestive of UBM from breast cancer or having symptoms. This is because of a missed examination of breast masses or the absence of early diagnosis programs.

UBM from breast cancer may present from one month to 30 years after the initial diagnosis of the primary tumor. Simultaneous presentation of UBM and breast cancer was reported [3,24,30]. It is worth mentioning that rarely the diagnosis of breast cancer followed the diagnosis of UBM [11]. The mean time of UBM from the primary diagnosis of breast cancer is 90 months, as reported in a large series [11]. Following the diagnosis of UBM, transurethral resection of the bladder lesion should be undertaken to stop hematuria. This also facilitates the stenting of ureters in the case of ureter obstruction. Percutaneous nephrostomy can also be undertaken to normalize renal function [25].

A combination of endocrine therapy and chemotherapy is the mainstay of treatment for UBM from breast cancer. The response to endocrine therapy is superior in the luminal A group of patients when compared with other subgroups and prolongs the disease-free survival in the luminal A group [23]. The prognosis of $\mathrm{MBC}$ to urinary bladder is similar to that of any MBC. The average survival is 18 to 30 months [31]. However, there are reports stating UBM has a worse prognosis than bone metastasis [32]. The reason postulated is that symptomatic UBM from breast cancer is detected at a late stage. The mucosal breach leads to gross hematuria, leading to investigations [33]. The majority of UBMs from breast cancer presented as part of systemic disease, which impacts survival. The reports stated that solitary UBM had shown increased diseasefree survival after treatment $[11,14,19,23]$. The role of radiotherapy is its ability to control hematuria and local control of the disease [34]. 


\section{Conclusions}

UBMs from breast cancer are rare, yet there is an increase in reports of such metastasis over the last few years, owing to better imaging and diagnostic modalities. The majority of UBMs present as part of widespread metastatic disease. Most UBMs occur in invasive lobular cancer. In addition to asymptomatic presentations, most cases present with hematuria and voiding dysfunction. After diagnosis with imaging, cystoscopy should be part of the workup in the biopsy of the lesion and performing transurethral resection. If no lesion is seen in a suspected case of UBM from breast cancer, random biopsies should be warranted. Chemotherapy and hormonal therapy are the mainstays of management, with radiotherapy being used to control bleeding. The prognosis of UBM is poor unless UBM represents the only metastatic site. Hence, patients with a history of breast cancer and urinary symptoms should be thoroughly evaluated for bladder metastasis.

\section{Additional Information \\ Disclosures}

Conflicts of interest: In compliance with the ICMJE uniform disclosure form, all authors declare the following: Payment/services info: All authors have declared that no financial support was received from any organization for the submitted work. Financial relationships: All authors have declared that they have no financial relationships at present or within the previous three years with any organizations that might have an interest in the submitted work. Other relationships: All authors have declared that there are no other relationships or activities that could appear to have influenced the submitted work.

\section{References}

1. Bray F, Ferlay J, Soerjomataram I, Siegel RL, Torre LA, Jemal A: Global cancer statistics 2018: GLOBOCAN estimates of incidence and mortality worldwide for 36 cancers in 185 countries. CA Cancer J Clin. 2018, 68:394-424. 10.3322/caac.21492

2. Karaosmanoglu AD, Onur MR, Karcaaltincaba M, Akata D, Ozmen MN: Secondary tumors of the urinary system: an imaging conundrum. Korean J Radiol. 2018, 19:742-751. 10.3348/kjr.2018.19.4.742

3. Junejo $\mathrm{N}, \mathrm{Al}$ Hussain $\mathrm{T}, \mathrm{Al}$ Hathal $\mathrm{N}$ : Unusual pattern of breast carcinoma metastasis to the urinary bladder: case report and review of literature. Saudi Surgical Journal. 2018, 6:66-68. 10.4103/ssj.ssj_4_18

4. Hess KR, Varadhachary GR, Taylor SH, Wei W, Raber MN, Lenzi R, Abbruzzese JL: Metastatic patterns in adenocarcinoma. Cancer. 2006, 106:1624-1633. 10.1002/cncr.21778

5. Wu Q, Li J, Zhu S, et al.: Breast cancer subtypes predict the preferential site of distant metastases a SEER based study. Oncotarget. 2017, 8:27990-27996. Accessed: March 24, 2020: 10.18632/oncotarget.15856

6. Abrams HL, Spiro R, Goldstein N: Metastases in carcinoma; analysis of 1000 autopsied cases . Cancer. 1950, 3:1-74. https://doi.org/10.1002/1097-0142(1950)3:1\%3C74::aid-cncr2820030111\%3E3.0.co;2-7

7. Klinger ME: Secondary tumors of the genito-urinary tract . J Urol. 1951, 65:144-153. 10.1016/s00225347(17)68470-2

8. Ganem EJ, Batal JT: Secondary malignant tumors of the urinary bladder metastatic from primary foci in distant organs. J Urol. 1956, 75:965-972. 10.1016/s0022-5347(17)66911-8

9. Goldstein AG: Metastatic carcinoma to the bladder . J Urol. 1967, 98:209-215. 10.1016/s0022-5347(17)62857$\mathrm{x}$

10. Pontes JE, Oldford JR: Metastatic breast carcinoma to the bladder. J Urol. 1970, 104:839-842. 10.1016/s00225347(17)61848-2

11. Feldman PA, Madeb R, Naroditsky I, Halachmi S, Nativ O: Metastatic breast cancer to the bladder: a diagnostic challenge and review of the literature. Urology. 2002, 59:138. 10.1016/s0090-4295(01)01489-3

12. Makki J: Diversity of breast carcinoma: histological subtypes and clinical relevance . Clin Med Insights Pathol. 2015, 8:23-31. Accessed: March 24, 2020: 10.4137/CPath.S31563

13. Borst MJ, Ingold JA: Metastatic patterns of invasive lobular versus invasive ductal carcinoma of the breast . Surgery. 1993, 114:637-641.

14. Ryan PD, Harisinghani M, Lerwill MF, Kaufman DS: Case records of the Massachusetts General Hospital. Case 6-2006. A 71-year-old woman with urinary incontinence and a mass in the bladder. N Engl J Med. 2006, 354:850-856. 10.1056/NEJMcpc059042

15. Ferlicot S, Vincent-Salomon A, Médioni J, et al.: Wide metastatic spreading in infiltrating lobular carcinoma of the breast. Eur J Cancer. 2004, 40:336-341. 10.1016/j.ejca.2003.08.007

16. Sherlock P, Hartmann WH: Adrenal steroids and the pattern of metastases of breast cancer . Jama. 1962, 181:313-317. 10.1001/jama.1962.03050300033007

17. Perou CM, Sørlie T, Eisen MB, et al.: Molecular portraits of human breast tumours. Nature. 2000, 406:747752. 10.1038/35021093

18. Elia G, Stewart S, Makhuli ZN, Krenzer BE, Mathur S, Simon HM, Mehdi S: Metastatic breast cancer diagnosed during a work-up for urinary incontinence: a case report. Int Urogynecol J Pelvic Floor Dysfunct. 1999, 10:39-42. 10.1007/pl00004013

19. Zagha RM, Hamawy KJ: Solitary breast cancer metastasis to the bladder: an unusual occurrence. Urol Oncol. 2007, 25:236-239. 10.1016/j.urolonc.2006.05.013

20. Iguchi C, Nio Y, Itakura M: Heterogeneic expression of estrogen receptor between the primary tumor and the corresponding involved lymph nodes in patients with node-positive breast cancer and its implications in patient outcome. J Surg Oncol. 2003, 83:85-93. 10.1002/jso.10243

21. Soon PS, Lynch W, Schwartz P: Breast cancer presenting initially with urinary incontinence: a case of bladder metastasis from breast cancer. Breast. 2004, 13:69-71. 10.1016/j.breast.2003.09.005

22. Ines Z, Riadh C, Hatem B, et al.: A case of solitary bladder metastasis from male breast cancer . Pan Arab J 


\section{Cureus}

Oncol. 2011, 4:8-9.

23. Lin WC, Chen JH: Urinary bladder metastasis from breast cancer with heterogeneic expression of estrogen and progesterone receptors. J Clin Oncol. 2007, 25:4308-4310. 10.1200/jco.2007.12.9379

24. Mairy Y, Opsomer R, Donnez J, Van Cangh PJ: [Bladder metastases from breast cancer: 2 cases] . Acta Urol Belg. 1982, 50:87-90.

25. Shah K, Modi P, Rizvi J: Breast carcinoma metastasizing to the urinary bladder and retroperitoneum presenting as acute renal failure. Indian J Urol. 2011, 27:135-136. 10.4103/0970-1591.78421

26. Balachandran AA, Duckett J: Metastatic breast cancer presenting as detrusor overactivity. BMJ Case Rep. 2014, 2014:Accessed: March 24, 2020: 10.1136/bcr-2014-207920

27. Al Ibraheemi AA: Case report of metastatic invasive breast lobular carcinoma to the urinary bladder . Int J Hematol Oncol Stem Cell Res. 2016, 10:51-55.

28. Wong MH, Yiu MK, Ho KL: Metastatic carcinoma of breast in the urinary bladder . Hong Kong Med J. 2013, 19:455-457. 10.12809/hkmj133768

29. Vranjesevic D, Filmont JE, Meta J, et al.: Whole-body (18)F-FDG PET and conventional imaging for predicting outcome in previously treated breast cancer patients. J Nucl Med. 2002, 43:325-329.

30. Cormio L, Sanguedolce F, Di Fino G, et al.: Asymptomatic bladder metastasis from breast cancer . Case Rep Urol. 2014, 2014:672591. Accessed: March 24, 2020: 10.1155/2014/672591

31. Gennari A, Conte P, Rosso R, Orlandini C, Bruzzi P: Survival of metastatic breast carcinoma patients over a 20-year period: a retrospective analysis based on individual patient data from six consecutive studies. Cancer. 2005, 104:1742-1750. 10.1002/cncr.21359

32. Lawrentschuk N, Chan Y, Bolton DM: Metastatic breast cancer to the bladder . Breast J. 2005, 11:143. 10.1111/j.1075-122X.2005.21427.x

33. Ghaida RA, Ayoub H, Nasr R, Issa G, Bulbul M: Bladder metastasis from primary breast cancer: a case report and literature review. Cent European J Urol. 2013, 66:177-184.

34. Din OS, Thanvi N, Ferguson CJ, Kirkbride P: Palliative prostate radiotherapy for symptomatic advanced prostate cancer. Radiother Oncol. 2009, 93:192-196. 10.1016/j.radonc.2009.04.017 